\title{
Diffraction on Nuclei: Effects of Nucleon Correlations
}

\author{
M. Alvioli \\ 104 Davey Lab., The Pennsylvania State University, University Park, PA 16803, USA \\ C. Ciofi degli Atti \\ Department of Physics, University of Perugia \\ and \\ Istituto Nazionale di Fisica Nucleare, Sezione di Perugia \\ Perugia, Via A. Pascoli, I-06123, Italy
}

\author{
B. Z. Kopeliovich, I. K. Potashnikova, and Iván Schmidt \\ Departamento de Física, Centro de Estudios Subatómicos, \\ Universidad Técnica Federico Santa María, \\ and \\ Centro Científico-Tecnológico de Valparaíso \\ Casilla 110-V, Valparaíso, Chile
}

(Dated: April 3, 2022)

\begin{abstract}
The cross sections for a variety of diffractive processes in proton-nucleus scattering, associated with large gaps in rapidity, are calculated within an improved Glauber-Gribov theory, where the inelastic shadowing corrections are summed to all orders by employing the dipole representation. The effects of nucleon correlations, leading to a modification of the nuclear thickness function, are also taken into account. Numerical calculations are performed for the energies of the HERA-B experiment, and the RHIC and LHC colliders, and for several nuclei. It is found that whereas the Gribov corrections generally make nuclear matter more transparent, nucleon correlations act in the opposite direction and have important effects in various diffractive processes.
\end{abstract}

PACS numbers: 24.85.+p, 13.85.Lg, 13.85.Lg, 25.55.Ci

\section{INTRODUCTION}

In hadron-nucleus collisions at high energies nuclei act almost like "black" absorbers. Therefore the optical analogy should be relevant and diffraction appears to be an important process. Experimentally diffraction appears as large rapidity gap events, when the debris of the projectile hadron and the nucleus occupy only small rapidity intervals close to the rapidities of the colliding particles. The optical analogy is employed by the Glauber theory [1] of hadron-nucleus interactions, which assumes additivity of the scattering phases on different bound nucleons. This is a single channel approximation assuming that absorption, i.e. inelastic interactions, generates via the unitarity relation only elastic scattering. In reality, diffractive excitations of hadrons frequently happen, and the Glauber approach was generalized to a multichannel case by Gribov [2]. The corresponding corrections to the Glauber approximation are known as inelastic shadowing, or Gribov corrections. Unfortunately, the multi-channel problem needs detailed experimental information, which is mostly unknown. One has to know all diffractive amplitudes, diagonal and off-diagonal, for different diffractive excitations of the hadron. Even the lowest order correction contains an unknown attenuation factor for an excited state propagating through the nucleus [3].

One can sum up the Gribov corrections to all orders by switching to the interaction eigenstates [4], which were identified in 5] as color dipoles, and where the dipole approach to high energy collisions was proposed. This phenomenology needs lesser input and the key ingredient, the dipole-nucleon cross section, is flavor independent and can be studied in different processes.

This method can be applied also to lepton- or photonnucleus collisions [6, 7, 8], where leptons and photons display hadronic properties. A detailed study of the inelastic shadowing corrections to different diffractive channels in proton-nucleus collisions was performed, within the dipole approach, in [9, 10].

Here we are going to enhance the accuracy of the calculations presented in 10], by improving the model for the nuclear wave function. Namely, most of calculations for nuclear shadowing effects have relied so far on a simplified model of an uncorrelated single particle density distribution in the nucleus. This model in particular ignores the well known experimental evidences for the existence of a strong repulsion core between nucleons. Such a repulsion should lead to short-range $N N$ correlations in the nuclear density function, which in turn should modify the effective nuclear thickness function controlling diffractive processes.

The consideration of possible effects from nucleonnucleon (NN) short range correlations (SRC) appears to be particularly interesting, in view of recent experimental data on lepton and hadron scattering off nuclei at medium energy, which provided quantitative evidence on SRC and their possible effects on dense hadronic matter 11]. Moreover, a recent calculation of the total neutron- 
nucleus cross section at Fermilab energies has indeed shown relevant effects from SRC even at high energies [12].

\section{GLAUBER FORMALISM}

The key assumption of the Glauber model is that the hadron-nucleus partial elastic amplitude at impact parameter $b$ has the eikonal form [1],

$$
\Gamma^{p A}\left(\vec{b} ;\left\{\vec{l}_{j}, z_{j}\right\}\right)=1-\prod_{k=1}^{A}\left[1-\Gamma^{p N}\left(\vec{b}-\vec{l}_{k}\right)\right]
$$

where $\left\{\vec{l}_{j}, z_{j}\right\}$ denote the coordinates of an $i$-th target nucleon; $i \Gamma^{p N}$ is the elastic scattering amplitude on a nucleon normalized as

$$
\sigma_{t o t}^{p N}=2 \int d^{2} b \operatorname{Re} \Gamma^{p N}(b)
$$

Further, one should calculate the matrix element of the amplitude (1) with the nuclear wave function, $\psi_{0}\left(\vec{r}_{1}, \vec{r}_{2}, \ldots, \vec{r}_{A}\right)=\psi_{0}\left(\left\{\vec{r}_{j}\right\}\right) \equiv|0\rangle$. Here we introduce new notations,

$G_{A}(\vec{b})=\left\langle 0\left|\Gamma^{p A}\left(\vec{b} ;\left\{\vec{l}_{j}, z_{j}\right\}\right)\right| 0\right\rangle=1-\left\langle 0\left|\prod_{i=1}^{A} G^{p N}\left(\vec{b}-\vec{l}_{i}\right)\right| 0\right\rangle$

where

$$
G^{p N}\left(\vec{b}-\vec{l}_{i}\right)=1-\Gamma^{p N}\left(\vec{b}-\vec{l}_{i}\right)
$$

The main problem in evaluating nuclear effects is therefore the choice of the nuclear wave function. $\psi_{0}(1, \ldots, A)$.

\section{A. Single particle approximation for the nuclear wave function}

The most popular model for the square of the nuclear wave function appearing in the Glauber formalism is the approximation of single particle nuclear density [36],

$$
\left|\psi_{o}\left(\vec{r}_{1}, \ldots, \vec{r}_{A}\right)\right|^{2} \simeq \prod_{j=1}^{A} \rho_{A}\left(\vec{b}_{1}, z_{1}\right)
$$

where

$$
\rho_{A}\left(\vec{b}_{1}, z_{1}\right)=\int \prod_{i=2}^{A} d^{3} r_{i}\left|\Psi_{A}\left(\left\{\vec{r}_{j}\right\}\right)\right|^{2}
$$

Within such an approximation the matrix element between the nuclear ground states reads,

$$
\begin{aligned}
& \left\langle 0\left|\Gamma^{p A}\left(\vec{b} ;\left\{\vec{l}_{j}, z_{j}\right\}\right)\right| 0\right\rangle \\
= & 1-\left[1-\frac{1}{A} \int d^{2} l \Gamma^{p N}(l) \int_{-\infty}^{\infty} d z \rho_{A}(\vec{b}-\vec{l}, z)\right]^{A} .
\end{aligned}
$$

Correspondingly, the total $p A$ cross section has the form,

$$
\begin{aligned}
\sigma_{\text {tot }}^{p A} & =2 \operatorname{Re} \int d^{2} b\left\{1-\left[1-\frac{1}{A} \int d^{2} l \Gamma^{p N}(l) T_{A}(\vec{b}-\vec{l})\right]^{A}\right\} \approx \\
& \approx 2 \int d^{2} b \times\left\{1-\exp \left[-\frac{1}{2} \sigma_{\text {tot }}^{p N}\left(1-i \alpha_{p N}\right) T_{A}^{h}(b)\right]\right\}
\end{aligned}
$$

where $\alpha_{p N}$ is the ratio of the real to imaginary parts of the forward $p N$ elastic amplitude;

$$
T_{A}^{h}(b)=\frac{2}{\sigma_{t o t}^{p N}} \int d^{2} l \operatorname{Re} \Gamma^{p N}(l) T_{A}(\vec{b}-\vec{l})
$$

and

$$
T_{A}(b)=\int_{-\infty}^{\infty} d z \rho_{A}(b, z)
$$

is the nuclear thickness function. We use the Gaussian form of $\Gamma^{p N}(l)$,

$$
\operatorname{Re} \Gamma^{p N}(l)=\frac{\sigma_{t o t}^{p N}}{4 \pi B_{e l}^{p N}} \exp \left(\frac{-l^{2}}{2 B_{e l}^{p N}}\right) .
$$

Notice that in Eq. (8) and in what follows we use the exponential approximation of large $A$ only to simplify and clarify the formulas. For numerical calculations throughout the paper we always rely on the exact expressions, such as the first part of Eq. (8).

The Glauber approach is a single channel model, therefore it is unable to consider diffractive excitation of the proton. However, a part of diffractive excitation of the nucleus occurs without excitation of the bound nucleons, when the nucleus just breaks up into free nucleons and nuclear fragments. Such events, $p A \rightarrow p F$, are called quasielastic and can be calculated within the Glauber approximation. Summing up the final states of the nucleus $|F\rangle$, applying the condition of completeness, and extract- 
ing the contribution of the ground state of the nucleus, one gets,

$$
\begin{aligned}
\sigma_{q e l}^{p A} & \equiv \sum_{F} \sigma(p A \rightarrow p F)-\sigma_{e l}^{p A}= \\
& =\sum_{F} \int d^{2} b\left[\left\langle 0\left|\Gamma^{p A}(b)\right| F\right\rangle^{\dagger}\left\langle F\left|\Gamma^{p A}(b)\right| 0\right\rangle-\left|\left\langle 0\left|\Gamma^{p A}(b)\right| 0\right\rangle\right|^{2}\right] \\
& =\int d^{2} b\left[\left\langle\left. 0|| \Gamma^{p A}(b)\right|^{2} \mid 0\right\rangle-\left|\left\langle 0\left|\Gamma^{p A}(b)\right| 0\right\rangle\right|^{2}\right] .
\end{aligned}
$$

In the first order in nuclear density the first term in this expression, $\left\langle\left. 0|| \Gamma^{p A}(b)\right|^{2} \mid 0\right\rangle$, contains, besides the usual linear term Eq. (99), the quadratic term $\int d^{2} s T_{A}(\vec{b}-$
$\overrightarrow{l)}\left[\Gamma^{p N}(l)\right]^{2}=T_{A}^{h}(b) \sigma_{e l}^{p N}$. Both terms together result in the exponent $\sigma_{i n}^{p N} T_{A}(b)$. Then the quasielastic cross section gets the form,

$$
\sigma_{q e l}^{p A}\left(p A \rightarrow p A^{*}\right)=\int d^{2} b\left\{\exp \left[-\sigma_{i n}^{p N} T_{A}^{h}(b)\right]-\exp \left[-\sigma_{t o t}^{p N} T_{A}^{h}(b)\right]\right\} .
$$

\section{B. Nucleon correlations}

Equation (5) represents only the lowest order term of the square of the full nuclear wave function $\left|\psi_{0}\right|^{2}$. As a matter of fact, the latter can be written as an expansion in terms of density matrices [1, 13] as follows:

$$
\left|\psi_{o}\left(\vec{r}_{1}, \ldots, \vec{r}_{A}\right)\right|^{2}=\prod_{j=1}^{A} \rho_{1}\left(\vec{r}_{j}\right)+\sum_{i<j} \Delta\left(\vec{r}_{i}, \vec{r}_{j}\right) \prod_{k \neq i, j} \rho_{1}\left(\vec{r}_{k}\right)+\sum_{(i<j) \neq(k<l)} \Delta\left(\vec{r}_{i}, \vec{r}_{j}\right) \Delta\left(\vec{r}_{k}, \vec{r}_{l}\right) \prod_{m \neq i, j, k, l} \rho_{1}\left(\vec{r}_{m}\right)+\ldots
$$

in which the single particle density $\rho_{1}\left(\vec{r}_{i}\right)$ is

$$
\rho_{1}\left(\vec{r}_{1}\right)=\int\left|\psi_{o}\left(\vec{r}_{1}, \vec{r}_{2}, \ldots, \vec{r}_{A}\right)\right|^{2} \prod_{i=2}^{A} d^{3} r_{i}
$$

and the two-body contraction $\Delta$ is

$$
\Delta\left(\vec{r}_{i}, \vec{r}_{j}\right)=\rho_{2}\left(\vec{r}_{i}, \vec{r}_{j}\right)-\rho_{1}\left(\vec{r}_{i}\right) \rho_{1}\left(\vec{r}_{j}\right) .
$$

The two-body density matrix

$$
\rho_{2}\left(\vec{r}_{1}, \vec{r}_{2}\right)=\int\left|\psi_{o}\left(\vec{r}_{1}, \vec{r}_{2}, \ldots, \vec{r}_{A}\right)\right|^{2} \prod_{i=3}^{A} d^{3} r_{i}
$$

satisfies the sequential condition,

$$
\int d^{3} r_{j} \rho_{2}\left(\vec{r}_{i}, \vec{r}_{j}\right)=\rho_{1}\left(\vec{r}_{i}\right)
$$

which leads to the basic property of the two-body contraction

$$
\int d^{3} r_{j} \Delta\left(\vec{r}_{i}, \vec{r}_{j}\right)=0
$$

Notice that the single particle density appearing in Eq. (14) is normalized to one, so that the densities defined by Eq. (6) and Eq. (15) are simply related by $\rho_{A}(\vec{r})=A \rho_{1}(\vec{r})$. It should be stressed that in Eq. (14) only unlinked contractions have to be considered, and that the higher order terms, not explicitly displayed, include unlinked products of 3,4 , etc two-body contractions, representing contributions to two-nucleon correlations, and unlinked products of three-body, four-body, etc, contractions, describing three-nucleon, four-nucleon, etc correlations. We will give now a short derivation of the total cross section including two-nucleon correlations 
(more details will be given elsewhere 14]). Taking into account all terms of the expansion (14) containing all possible numbers of unlinked two-body contractions, Eq.
(3) can be written in the following form which yields the usual Glauber profile when $\Delta=0[12,14]$ :

$$
\begin{aligned}
& G_{A}(b) \equiv \int \prod_{k=1}^{A} d^{3} r_{k}|\psi(1 \ldots A)|^{2} \prod_{i=1}^{A} G^{p N}\left(\vec{b}-\vec{l}_{i}\right)= \\
& \quad=\int \prod_{k=1}^{A} d^{3} r_{k} \rho_{1}\left(\vec{r}_{k}\right) G^{p N}\left(\vec{b}-\vec{l}_{k}\right)+\sum_{i<j} \int \prod_{k=1}^{A} d^{3} r_{k} \Delta\left(\vec{r}_{i}, \vec{r}_{j}\right) \prod_{l \neq i, j}^{A} \rho_{1}\left(\vec{r}_{l}\right) G^{p N}\left(\vec{b}-\vec{l}_{k}\right)+ \\
& \quad+\sum_{i<j \neq p<l} \int \prod_{k=1}^{A} d^{3} r_{k} \Delta\left(\vec{r}_{i}, \vec{r}_{j}\right) \Delta\left(\vec{r}_{p}, \vec{r}_{l}\right) \prod_{m \neq i, j, p, l}^{A} \rho_{1}\left(\vec{r}_{m}\right) G^{p N}\left(\vec{b}-\vec{l}_{k}\right)+\cdots \\
& =G_{A}^{(0)}(\vec{b})+G_{A}^{(1)}(\vec{b})+G_{A}^{(2)}(\vec{b})+\cdots
\end{aligned}
$$

where the superscript denotes the number of two-body contractions in the given term, each term containing Glauber profiles to all orders. For each nucleus we have considered all terms of the series (20); the first term, corresponding to the single particle approximation of Eq. (5) being

$$
\begin{aligned}
G_{A}^{(0)}(b) & =\int \prod_{k=1}^{A} d^{3} r_{k} \rho_{1}\left(\vec{r}_{k}\right) G^{p N}\left(\vec{b}-\vec{l}_{k}\right) \\
& =\left[1-\frac{1}{A} \int d^{3} r_{1} \rho_{A}\left(\vec{r}_{1}\right) \Gamma^{p N}\left(\vec{b}-\vec{l}_{1}\right)\right]^{A}
\end{aligned}
$$

and the n-th terms

$$
G_{A}^{(n)}(b)=\frac{A !}{2^{n} n !(A-2 n) !} X^{n}(b) Y^{A-2 n}(b)
$$

where

$$
X(b)=\int d^{3} r_{1} d^{3} r_{2} \Delta\left(\vec{r}_{1}, \vec{r}_{2}\right) \Gamma^{p N}\left(\vec{b}-\vec{l}_{1}\right) \Gamma^{p N}\left(\vec{b}-\vec{l}_{2}\right)
$$

and

$$
Y(b)=\left[1-\frac{1}{A} \int d^{3} r_{1} \rho_{A}\left(\vec{r}_{1}\right) \Gamma^{p N}\left(\vec{b}-\vec{b}_{1}\right)\right]
$$

resulting from the basic properties of the two-body contraction: $\int d^{3} r_{i, j} \Delta\left(\vec{r}_{i}, \vec{r}_{j}\right)=0$ and

$$
\int d^{3} r_{1} d^{3} r_{2} \Delta\left(\vec{r}_{1}, \vec{r}_{2}\right) G^{p N}\left(\vec{b}-\vec{l}_{1}\right) G^{p N}\left(\vec{b}-\vec{l}_{2}\right)=\int d^{3} r_{1} d^{3} r_{2} \Delta\left(\vec{r}_{1}, \vec{r}_{2}\right) \Gamma^{p N}\left(\vec{b}-\vec{l}_{1}\right) \Gamma^{p N}\left(\vec{b}-\vec{l}_{2}\right)
$$

Eq. (20) can now be written as follows

$$
G_{A}(b)=\sum_{n=0}^{A / 2} \frac{A ! X^{n}(b)[Y(b)]^{A-2 n}}{2^{n} n !(A-2 n) !} \underset{A \gg 1}{\longrightarrow}[Y(b)]^{A} \sum_{n=0}^{\infty} \frac{A^{2 n} X^{n}(b)}{2^{n} n !}=[Y(b)]^{A} e^{\frac{A^{2}}{2} X(b)} .
$$

Using, for ease of presentation, the optical limit approximation 


$$
[Y(b)]^{A}=\left[1-\frac{1}{A} \int d^{3} r_{1} \rho_{1}\left(\vec{r}_{1}\right) \Gamma^{p N}\left(\vec{b}-\vec{l}_{1}\right)\right]^{A}=e^{-\int d^{3} r_{1} \rho_{A}\left(\vec{r}_{1}\right) \Gamma\left(\vec{b}-\vec{l}_{1}\right)},
$$

the insertion of Eq. (27) into Eq. (26) leads to the final result:

$$
\begin{aligned}
G_{A}(b) & \simeq 1-\exp \left[-\int d^{3} r_{1} \rho_{A}\left(\vec{r}_{1}\right) \Gamma\left(\vec{b}-\vec{l}_{1}\right)+\frac{1}{2} \int d^{3} r_{1} d^{3} r_{2} \Delta_{A}\left(\vec{r}_{1}, \vec{r}_{2}\right) \Gamma\left(\vec{b}-\vec{l}_{1}\right) \Gamma\left(\vec{b}-\vec{l}_{2}\right)\right]= \\
& =1-\exp \left[-\frac{1}{2} \sigma_{t o t}^{p N} \widetilde{T}_{A}^{h}(b)\right]
\end{aligned}
$$

where

$$
\Delta_{A}\left(\vec{r}_{1}, \vec{r}_{2}\right)=\rho_{A}^{(2)}\left(\vec{r}_{1}, \vec{r}_{2}\right)-\rho_{A}\left(\vec{r}_{1}\right) \rho_{A}\left(\vec{r}_{2}\right) .
$$

which obviously differs by Eq. (16) simply by a factor $A^{2}$, and

$$
\widetilde{T}_{A}^{h}(b)=T_{A}^{h}(b)-\Delta T_{A}^{h}(b),
$$

with $T_{A}^{h}(b)$ given by Eq. (9) and

$$
\begin{aligned}
\Delta T_{A}^{h}(b) & =\frac{\left(1-i \alpha_{p N}\right)}{\sigma_{t o t}^{p N}} \int d^{2} l_{1} d^{2} l_{2} \Delta_{A}^{\perp}\left(\vec{l}_{1}, \vec{l}_{2}\right) \\
& \times \operatorname{Re} \Gamma^{p N}\left(\vec{b}-\vec{l}_{1}\right) \operatorname{Re} \Gamma^{p N}\left(\vec{b}-\vec{l}_{2}\right),
\end{aligned}
$$

where

$$
\Delta_{A}^{\perp}\left(\vec{l}_{1}, \vec{l}_{2}\right)=\int_{-\infty}^{\infty} d z_{1} \int_{-\infty}^{\infty} d z_{2} \Delta_{A}\left(\vec{r}_{1}, \vec{r}_{2}\right)
$$

is the transverse two-nucleon contraction. It can be seen that the inclusion of $N N$ correlations in nuclei leads to a modification of the nuclear thickness function $T_{A}^{h}(b) \Rightarrow \widetilde{T}_{A}^{h}(b)$. Due to its general structure and the basic property $\int d^{3} r_{1,2} \Delta_{A}\left(\vec{r}_{1}, \vec{r}_{2}\right)=0$, the sign of the contraction is mostly negative, with a small positive contribution at large separations. In Fig. 1 we present $T_{A}^{h}(b)$ and $\Delta T_{A}^{h}(b)$ for ${ }^{12} C$ and ${ }^{208} P b$. We see that $\Delta T_{A}^{h}(b)$ is indeed mostly negative, so according to the definition Eq. (30) correlations increase the nuclear thickness function and make nuclear medium more opaque 12 . At the same time, the corrections are small, $\Delta T_{A}^{h}(b) \ll T_{A}^{h}(b)$, and the effects from higher order correlations, estimated in Ref. [12], can safely be disregarded.

A short description of the way in which the one and two-body densities and contractions have been calculated is now in order. Following Ref. [12]. the twobody density has been obtained from the fully-correlated wave function of Ref. [15, 16], $\psi_{0}=\hat{F} \phi_{0}$, where $\hat{F}=$ $\prod_{i<j}\left[\sum_{k=1}^{8} f_{k}\left(r_{i j}\right) \hat{O}_{k}(i j)\right]$ is a correlation operator generated by the realistic Argonne $V 8^{\prime}$ interaction [17], and $\phi_{0}$ a mean field shell model wave function composed of Woods-Saxon single particle orbitals. The above wave function largely differs from the Jastrow one, featuring only central correlations, since the operator $\hat{F}$ generates central $\left(\hat{O}_{1}=1\right)$, spin $\left(\hat{O}_{2}(i j)=\vec{\sigma}_{i} \cdot \vec{\sigma}_{j}\right)$, isospin $\left(\hat{O}_{3}(i j)=\right.$ $\left.\vec{\tau}_{i} \cdot \vec{\tau}_{j}\right)$, spin-isospin $\left(\hat{O}_{4}(i j)=\left(\vec{\sigma}_{i} \cdot \vec{\sigma}_{j}\right)\left(\vec{\tau}_{i} \cdot \vec{\tau}_{j}\right)\right)$, tensor $\left(\hat{O}_{5}(i j)=\vec{S}_{i j}\right)$, tensor-isospin $\left(\hat{O}_{6}(i j)=\vec{S}_{i j}\left(\vec{\tau}_{i} \cdot \vec{\tau}_{j}\right)\right)$, etc. correlations. The two-body density and contraction therefore reflect not only the short range repulsion but also the spin-isospin dependence of the interaction, particularly that generated by the tensor force. The parameters of both the single particle wave functions and the various correlation functions have been fixed from the ground- state energy calculation so that no free parameters are present in our approach.

The contraction $\Delta\left(\vec{r}_{1}, \vec{r}_{2}\right)$ resulting from our calculation exactly satisfies the sum rule $\int d^{3} r_{1} \Delta\left(\overrightarrow{r_{1}}, \vec{r}_{2}\right)=0$, since the one-body density $\rho_{1}\left(\vec{r}_{1}\right)$ exactly results from the integration of $\rho_{2}\left(\vec{r}_{1}, \vec{r}_{2}\right)$. Notice, moreover, that our onebody point density and radii are in agreement with electron scattering data [18]. We have also investigated the validity of the approximation in which the nuclear matter two-body density $\rho_{2}\left(\vec{r}_{1}, \vec{r}_{2}\right)=\rho_{1}\left(\vec{r}_{1}\right) \rho_{1}\left(\vec{r}_{2}\right) g\left(\left|\vec{r}_{1}-\vec{r}_{2}\right|\right)$ is used for finite nuclei, finding that it leads to a strong violation of the sequential relation $\int d^{3} r \rho_{2}\left(\vec{r}_{1}, \vec{r}_{2}\right)=\rho_{1}\left(\vec{r}_{1}\right)$ for nuclei with $A<208$. Thus, when such an approximation is used to introduce correlations in light and medium-weight nuclei, a mismatch between the one-body density (usually taken from the experimental data) and the two-body density is generated.

Using the nuclear thickness function which includes the effects of correlations, Eq. (30), the total cross section, Eq. (8), acquires a correction, $\sigma_{\text {tot }}^{p A} \Rightarrow \sigma_{\text {tot }}^{p A}+\Delta \sigma_{\text {tot }}^{p A}$, which 
is positive and can be approximated as,

$$
\Delta \sigma_{\text {tot }}^{p A} \approx-\sigma_{\text {tot }}^{p N} \int d^{2} b \Delta T_{A}^{h}(b) \exp \left[-\frac{1}{2} \sigma_{t o t}^{p N} T_{A}^{h}(b)\right] .
$$

which is also positive, since $\Delta T_{A}^{h}(b)$ is itself negative.

We see that this correction to the total cross section comes mainly from peripheral collisions, and rises with $A$ rather slowly, as $A^{1 / 3}$. Notice that the accuracy of the

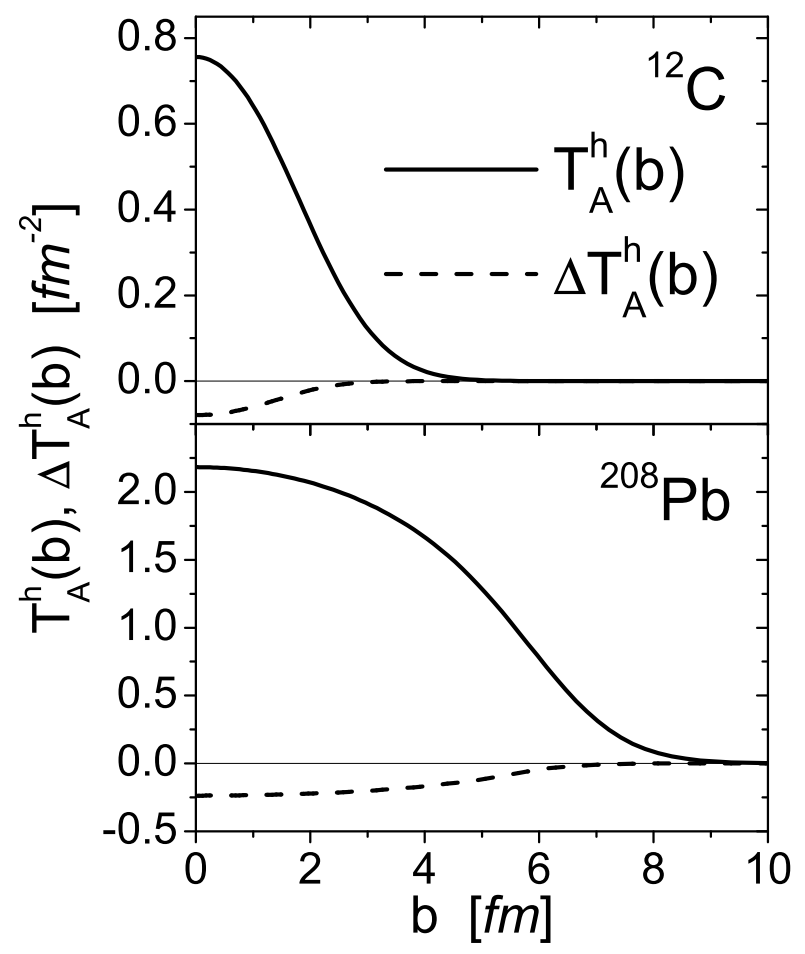

FIG. 1: Nuclear thickness function $T_{A}^{h}(b)$ and the correction due to $N N$-correlations, $\Delta T_{A}^{h}(b)$, calculated at the energy of HERA-B, for carbon and lead, respectively.

optical (exponential) approximation in (8) is quite good, $\sim 10^{-3}$ for heavy nuclei, but it gets worse with decreasing $A$, therefore for numerical calculations, as was already mentioned, we rely upon the exact Glauber expressions throughout the paper. In what follows we neglect the real part of the elastic amplitude which gives quite a small correction, $\sim \rho_{p p}^{2} / A^{2 / 3}$, and which otherwise can be easily implemented.

The simplest process with a Large Rapidity Gap (LRG) is elastic scattering. It is worth noting, however, that this channel is enhanced by absorptive corrections, while other LRG processes considered below are suppressed by these corrections.

The elastic cross section according to (7) reads,

$$
\sigma_{e l}^{p A}=\int d^{2} b\left|1-\exp \left[-\frac{1}{2} \sigma_{t o t}^{p N} \widetilde{T}_{A}^{h}(b)\right]\right|^{2},
$$

where $\widetilde{T}_{A}^{h}(b)$ is given by (30).

The quasielastic cross section also gets modifications compared to the Glauber expression Eq. (13). The nucleon correlations show up in the second order in nuclear density, leading to an additional term proportional to $\left(\sigma_{i n}^{p N}\right)^{2} \Delta T_{A}^{h}(b)$. Thus, the cross section of quasielastic proton-nucleus scattering, $p A \rightarrow p A^{*}$, gets the form,

$$
\begin{gathered}
\sigma_{q e l}^{p A}=\int d^{2} b\left\{\exp \left[-\sigma_{i n}^{p N} T_{A}^{h}(b)-\frac{\left(\sigma_{\text {in }}^{p N}\right)^{2}}{\sigma_{\text {tot }}^{p N}} \Delta T_{A}^{h}(b)\right]\right. \\
\left.-\exp \left[-\sigma_{\text {tot }}^{p N}\left(T_{A}^{h}(b)+\Delta T_{A}^{h}(b)\right)\right]\right\} .
\end{gathered}
$$

Notice that in deriving this expression we implicitly used the assumption that the impact parameter dependence of powers of the amplitude $\Gamma^{p N}(s)$ does not depend on the power. Although this is certainly not correct, the approximation is rather accurate as far as the $N N$ interaction radius is much smaller than the size of the nucleus. Nevertheless, we used this approximation only for the sake of clarity and simplicity. For numerical calculations, we use the more complicated but exact analogue of Eq. (35).

\section{GRIBOV CORRECTIONS VIA LIGHT-CONE DIPOLES}

The dipole representation for the amplitude of hadronic interactions allows to sum up the Gribov inelastic corrections to all order. We assume the collision energy to be high enough to keep the dipole size "frozen" by Lorentz time delation during propagation through the nucleus. In this limit the calculations are much simplified.

The key ingredients of the approach are the universal dipole-nucleon cross section and the light-cone wave function of the projectile hadron [5]. Several different models were tested in [10], by comparing with data on proton diffraction. Here we select two models which describe diffraction quite well. Both employ the saturated shape of the dipole cross section and differ only by modeling the proton wave function.

In the limit of soft interactions the Bjorken $x$ is not a proper variable any more, and the dipole cross section should depend on energy. We rely on the model proposed in [33] and fitted to data,

$$
\sigma_{\bar{q} q}\left(r_{T}, s\right)=\sigma_{0}(s)\left[1-\exp \left(-\frac{r_{T}^{2}}{R_{0}^{2}(s)}\right)\right],
$$

where $R_{0}(s)=0.88 \mathrm{fm}\left(s_{0} / \mathrm{s}\right)^{0.14}$ and $s_{0}=1000 \mathrm{GeV}^{2}$ 33]. The energy dependent factor $\sigma_{0}(s)$ is defined as,

$$
\sigma_{0}(s)=\sigma_{t o t}^{\pi p}(s)\left(1+\frac{3 R_{0}^{2}(s)}{8\left\langle r_{c h}^{2}\right\rangle_{\pi}}\right),
$$

where $\left\langle r_{c h}^{2}\right\rangle_{\pi}=0.44 \pm 0.01 \mathrm{fm}^{2}$ [19] is the mean square of the pion charge radius. This dipole cross section is nor- 
malized to reproduce the pion-proton total cross section, $\left\langle\sigma_{\bar{q} q}\right\rangle_{\pi}=\sigma_{\text {tot }}^{\pi p}(s)$.

For the proton wave function we employ two models.

\section{A. $q-2 q$ model}

There are many evidences (although neither of them looks decisive) for a strong paring of the $u$ and $d$ valence quarks into a small size scalar-isoscalar diquark [20, 21, 22]. Neglecting the diquark radius we arrive at a mesontype color dipole structure of the proton,

$$
\left|\Psi_{N}\left(\vec{r}_{1}, \vec{r}_{2}, \vec{r}_{3}\right)\right|^{2}=\frac{2}{\pi R_{p}^{2}} \exp \left(-\frac{2 r_{T}^{2}}{R_{p}^{2}}\right),
$$

where $\vec{r}_{i}$ are the interquark transverse distances, $\vec{r}_{3}=0$, $\vec{r}_{T}=\vec{r}_{1}=\vec{r}_{2}$, and $R_{p}$ is related to the mean charge radius squared of the proton as $R_{p}^{2}=\frac{16}{3}\left\langle r_{c h}^{2}\right\rangle_{p}$. The dipole wave function squared, Eq. (38), convoluted with the dipole cross section, Eq. (36), gives the proton-proton total cross section.

In this model the effect of the Gribov corrections in all orders is equivalent to the replacement of the Glauber formula Eq. (8) by,

$$
\begin{aligned}
\sigma_{\text {tot }}^{p A} & =2 \int d^{2} b \int_{0}^{1} d \alpha \int d^{2} r_{T}\left|\Psi_{N}\left(r_{T}, \alpha\right)\right|^{2} \\
& \times\left[1-e^{-\frac{1}{2} \sigma_{\bar{q} q}\left(r_{T}, s\right) T_{A}^{\bar{q} q}\left(b, r_{T}, \alpha\right)}\right] \\
& \equiv 2 \int d^{2} b\left[1-\left\langle e^{-\frac{1}{2} \sigma_{\bar{q} q}\left(r_{T}, s\right) T_{A}^{\bar{q} q}\left(b, r_{T}, \alpha\right)}\right\rangle\right] .
\end{aligned}
$$

Here we consider a $\bar{q} q$ (or $q q-q$ ) dipole of transverse separation $\vec{r}_{T}$ and fractional light-cone momenta $\alpha$ and $1-\alpha$ of the constituents. The integration over these variables weighted by the hadron wave function squared is denoted as averaging. The new notation $T_{A}^{\bar{q} q}\left(b, r_{T}, \alpha\right)$ is,

$T_{A}^{\bar{q} q}\left(b, r_{T}, \alpha\right)=\frac{2}{\sigma_{\bar{q} q}\left(r_{T}\right)} \int d^{2} l \operatorname{Re} \Gamma^{\bar{q} q N}\left(\vec{l}, \vec{r}_{T}, \alpha\right) T_{A}(\vec{b}-\vec{l})$.

The partial dipole-nucleon elastic amplitude $\operatorname{Re} \Gamma^{\bar{q} q N}\left(\vec{l}, r_{T}, \alpha\right)$, corresponding to the dipole cross section (36), was derived recently in [23, 24, 25],

$$
\begin{aligned}
& \operatorname{Re} \Gamma^{\bar{q} q N}\left(\vec{l}, \vec{r}_{T}, \alpha\right)=\frac{\sigma_{0}(s)}{8 \pi B(s)} \\
\times & \left\{\exp \left[-\frac{\left[\vec{l}+\vec{r}_{T}(1-\alpha)\right]^{2}}{2 B(s)}\right]+\exp \left[-\frac{\left(\vec{l}-\vec{r}_{T} \alpha\right)^{2}}{2 B(s)}\right]\right. \\
- & \left.2 \exp \left[-\frac{r_{T}^{2}}{R_{0}^{2}(s)}-\frac{\left[\vec{l}+(1 / 2-\alpha) \vec{r}_{T}\right]^{2}}{2 B(s)}\right]\right\}
\end{aligned}
$$

where $B(s)=B_{e l}^{p N}(s)-\frac{1}{3}\left\langle r_{c h}^{2}\right\rangle_{p}-\frac{1}{8} R_{0}^{2}(s)$. It is easy to check that this partial amplitude correctly reproduces the dipole-nucleon cross section Eq. (36),

$$
\sigma_{\bar{q} q}\left(r_{T}, s\right)=2 \int d^{2} l \operatorname{Re} \Gamma^{\bar{q} q N}\left(\vec{l}, \vec{r}_{T}, \alpha\right),
$$

and the slope of the differential elastic $p N$ scattering,

$$
B_{e l}^{p N}(s)=\frac{1}{\sigma_{t o t}^{p N}} \int d^{2} l l^{2}\left\langle\operatorname{Re} \Gamma^{\bar{q} q N}\left(\vec{l}, \vec{r}_{T}, \alpha\right)\right\rangle .
$$

These properties of the partial amplitude lead to the following relations with the analogous functions defined above within the Glauber model,

$$
\begin{aligned}
\sigma_{t o t}^{p N} & =\left\langle\sigma_{\bar{q} q}\left(r_{T}\right)\right\rangle \\
\operatorname{Re} \Gamma^{p N}(l) & =\left\langle\operatorname{Re} \Gamma^{\bar{q} q N}\left(\vec{l}, \vec{r}_{T}, \alpha\right)\right\rangle ; \\
T_{A}^{h}(b) & =\frac{1}{\sigma_{\text {tot }}^{p N}}\left\langle\sigma_{\bar{q} q}\left(r_{T}\right) T_{A}^{\bar{q} q}\left(b, r_{T}, \alpha\right)\right\rangle .
\end{aligned}
$$

Thus, the difference between the Glauber formula Eq. (8), and the exact expression, Eq. (39), is in how the averaging over $r_{T}$ and $\alpha$ is done: in the former case the averaging is done up in the exponent, while in the latter case the whole exponential is averaged.

Notice that $T_{A}^{\bar{q} q}\left(b, r_{T}, \alpha\right)$ in the exponent in Eq. (39) can be replaced by $T_{A}^{h}(b)$ with a high precision. Indeed, at small $r_{T}^{2} \ll R_{0}^{2}(s)$ the partial amplitude Eq. (41) vanishes as $\operatorname{Re} \Gamma^{\bar{q} q N}\left(\vec{l}, \vec{r}_{T}, \alpha\right) \propto r_{T}^{2}$. This $r_{T}$ dependence cancels in (40) with the same behavior of $\sigma_{\bar{q} q}\left(r_{T}\right)$ in the denominator. Thus, $T_{A}^{\bar{q} q}\left(b, r_{T}, \alpha\right)$ is independent of $r_{T}$ in this limit. In the opposite limit of large $r_{T}^{2} \gg R_{0}^{2}(s)$ the last term in (39) vanishes, and the amplitude integrated over $d^{2} l$ becomes a constant. Moreover, the denominator of Eq. (40) is independent of $r_{T}$ in this limit. Thus, one can neglect the slow $r_{T}$ dependence of $T_{A}^{\bar{q} q}\left(b, r_{T}, \alpha\right)$ in Eq. (46) in comparison with the fast varying function $\sigma_{\bar{q} q}\left(r_{T}\right)$, which is equivalent to the replacement $T_{A}^{\bar{q} q}\left(b, r_{T}, \alpha\right) \Rightarrow T_{A}^{h}(b)$. We rely on this approximation in Eq. (39) and in what follows.

Thus, for the total $p-A$ cross section we recover the standard expression [5, 10],

$$
\begin{aligned}
\sigma_{\text {tot }}^{p A} & =2 \int d^{2} b \int d^{2} r_{T}\left|\Psi_{N}\left(r_{T}\right)\right|^{2} \\
& \times\left\{1-\exp \left[-\frac{1}{2} \sigma_{\bar{q} q}\left(r_{T}, s\right) T_{A}^{h}(b)\right]\right\},
\end{aligned}
$$

\section{B. $3 q$ model}

Another extreme is to assume no pairing forces and a symmetric valence quark wave function,

$$
\begin{aligned}
& \left|\Psi_{N}\left(\vec{r}_{1}, \vec{r}_{2}, \vec{r}_{3}\right)\right|^{2}=\frac{3}{\left(\pi R_{p}^{2}\right)^{2}} \delta\left(\vec{r}_{1}+\vec{r}_{2}+\vec{r}_{3}\right), \\
\times & \exp \left(-\frac{r_{1}^{2}+r_{2}^{2}+r_{3}^{2}}{R_{p}^{2}}\right)
\end{aligned}
$$


Here the mean interquark separation squared is $\left\langle\vec{r}_{i}^{2}\right\rangle=$ $\frac{2}{3} R_{p}^{2}=2\left\langle r_{c h}^{2}\right\rangle_{p}$. In this case one needs a cross section for a

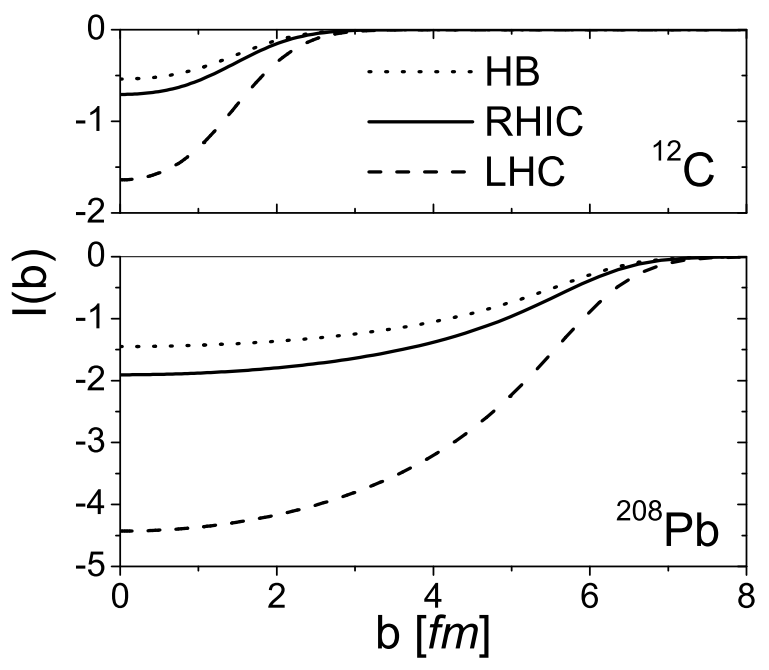

FIG. 2: The integral of Eq. (55) for carbon and lead, calculated at HERA B (dotted curves), RHIC (solid curves) and LHC (dashed curves) energies.

three-quark dipole, $\sigma_{3 q}\left(\vec{r}_{1}, \vec{r}_{2}, \vec{r}_{3}\right)$, where $\vec{r}_{i}$ are the transverse quark separation, with the condition $\vec{r}_{1}+\vec{r}_{2}+\vec{r}_{3}=0$. In order to avoid the introduction of a new unknown phenomenological quantity, we express the three-body dipole cross section via the conventional dipole cross section $\sigma_{\bar{q} q}$ [9, 10],

$$
\sigma_{3 q}\left(\vec{r}_{1}, \vec{r}_{2}, \vec{r}_{3}\right)=\frac{1}{2}\left[\sigma_{\bar{q} q}\left(r_{1}\right)+\sigma_{\bar{q} q}\left(r_{2}\right)+\sigma_{\bar{q} q}\left(r_{3}\right)\right] .
$$

This form satisfies the limiting conditions, namely, turns into $\sigma_{\bar{q} q}(r)$ if one of three separations is zero.

In this model the Gribov corrections modify the Glauber expression Eq. (8) as,

$$
\begin{gathered}
\sigma_{\text {tot }}^{p A}=2 \int d^{2} b \int d^{2} r_{1} d^{2} r_{2} d^{2} r_{3}\left|\Psi_{N}\left(r_{i}\right)\right|^{2} \\
\times\left\{1-\exp \left[-\frac{1}{2} \sigma_{3 q}\left(r_{i}, s\right) T_{A}^{h}(b)\right]\right\},
\end{gathered}
$$

\section{GRIBOV CORRECTIONS TO THE EFFECT OF $N N$ CORRELATIONS}

Nucleon correlations lead to further modifications of the exponent in Eq. (39), which correspond to the replacement $T_{A}^{\bar{q} q}\left(b, r_{T}, \alpha\right) \Rightarrow T_{A}^{\bar{q} q}\left(b, r_{T}, \alpha\right)+\Delta T_{A}^{\bar{q} q}(b)$, where

$$
\begin{gathered}
\Delta T_{A}^{\bar{q} q}\left(b, r_{T}, \alpha\right)=\frac{1}{\sigma_{\bar{q} q}\left(r_{T}\right)} \int d^{2} l_{1} d^{2} l_{2} \Delta_{A}^{\perp}\left(\vec{l}_{1}, \vec{l}_{2}\right) \\
\times \quad \operatorname{Re} \Gamma^{\bar{q} q}\left(\vec{b}-\vec{l}_{1}, r_{T}, \alpha\right) \operatorname{Re} \Gamma^{\bar{q} q}\left(\vec{b}-\vec{l}_{2}, r_{T}, \alpha\right),
\end{gathered}
$$

Changing the integration variables $d^{2} l_{1} d^{2} l_{2} \Rightarrow d^{2} L d^{2} \delta$, where

$$
\begin{aligned}
\vec{L} & =\left(\overrightarrow{l_{1}}+\overrightarrow{l_{2}}\right) / 2 ; \\
\vec{\delta} & =\vec{l}_{1}-\vec{l}_{2} ;
\end{aligned}
$$

one has, correspondingly, $\Delta_{A}^{\perp}\left(\vec{l}_{1}, \vec{l}_{2}\right) \Rightarrow \Delta_{A}^{\perp}(\vec{L}, \vec{\delta})$. This function is rather smooth and varies over distances much longer than the interaction radius. Therefore, we can take it out of the integral in Eq. (51), fixing it at $\vec{L}=$ $\vec{b}$. Then, using the partial amplitude Eq. (41) one can perform the integration over $d^{2} L$ in (51) and then average over $r_{T}$ and $\alpha$. The result is,

$$
\begin{aligned}
& \int d^{2} L\left\langle\operatorname{Re} \Gamma^{\bar{q} q}\left(\vec{l}_{1}, r_{T}, \alpha\right) \operatorname{Re} \Gamma^{\bar{q} q}\left(\vec{l}_{2}, r_{T}, \alpha\right)\right\rangle= \\
= & {\left[\sigma_{e l}^{p N}+\sigma_{s d}^{p N}\right] \exp \left[-\frac{\delta^{2}}{4 B(s)+R_{0}^{2}(s) / 2}\right] }
\end{aligned}
$$

Here $\sigma_{s d}^{p N}$ is the single diffraction cross section, $p N \rightarrow$ $X N$; and the relation [5, 10, 26.

$$
\int d^{2} L\left\langle\left[\operatorname{Re} \Gamma^{\bar{q} q}\left(\vec{L}, r_{T}, \alpha\right)\right]^{2}\right\rangle=\sigma_{e l}^{p N}+\sigma_{s d}^{p N} .
$$

has been used.

Data show that at high energies this cross section is nearly constant and is about $\sigma_{s d}^{p N} \approx 4 \mathrm{mb}$, the value which we use in what follows.

Notice that data for single diffraction also include the contribution from the triple-Pomeron term, which corresponds to diffractive gluon radiation. This term has not been included so far in our calculations, which correspond only to diffractive excitation of the valence quark skeleton of the proton (see in [10]). However, the higher Fock components of the light-cone wave function of the proton should be also added, which effectively incorporate this contribution by using the total single diffraction cross section.

Eq. (53) turns into the Glauber model relation Eq. (31) if the diffraction term is removed and the denominator of the exponent is replaced by $4 B_{e l}^{p N}$.

Eventually, the correction related to the nucleon correlations to the nuclear thickness function, convoluted with the dipole cross section, takes the form,

$$
\begin{gathered}
I_{A}(b)=\left\langle\sigma_{\bar{q} q}\left(r_{T}\right) \Delta T_{A}^{\bar{q} q}\left(b, r_{T}, \alpha\right)\right\rangle=\left[\sigma_{e l}^{p N}+\sigma_{s d}^{p N}\right] \\
\times \int d^{2} \delta \exp \left[-\frac{\delta^{2}}{4 B(s)+R_{0}^{2}(s) / 2}\right] \Delta_{A}^{\perp}(\vec{\delta}, b) .
\end{gathered}
$$

The quantity $I_{A}(b)$ is shown in Fig. 2. for both ${ }^{12} C$ and ${ }^{208} \mathrm{~Pb}$.

Since $\Delta T_{A}$ is small and its higher orders are negligible, there is no difference between averaging of the exponential and of its exponent. Therefore, the result Eq. 55) accounts for all inelastic shadowing effects in $N N$ correlations. Then, the total cross section reads,

$$
\sigma_{\text {tot }}^{p A}=2 \int d^{2} b\left\{1-e^{\frac{1}{2} I_{A}(b)}\left\langle e^{-\frac{1}{2} \sigma_{d i p} T_{A}^{h}(b)}\right\rangle\right\} .
$$


Here we use a notation which unifies the two models under consideration. $\sigma_{\text {dip }}$ is the dipole cross section, and averaging corresponds to integration over the light-cone momenta of the quarks, weighted with the proton wave function squared.

The results of the calculation of the total, elastic and quasi-elastic cross section for several nuclei and HERA B, RHIC and LHC energies, obtained within the Glauber approach including NN correlations, are presented in Tables I, II, III and IV.

TABLE I: HERA B.

\begin{tabular}{c|cccc}
\hline \hline${ }^{12} C$ & Glauber & $\begin{array}{c}\text { Glauber } \\
+ \text { SRC }\end{array}$ & $\begin{array}{c}\text { q-2q model } \\
+ \text { SRC }\end{array}$ & $\begin{array}{c}\text { 3q model } \\
+ \text { SRC }\end{array}$ \\
\hline$\sigma_{t o t}$ & 353.71 & 364.11 & 344.16 & 349.37 \\
\hline$\sigma_{e l}$ & 86.90 & 92.96 & 82.39 & 85.42 \\
\hline$\sigma_{s d}$ & - & - & 5.43 & 2.40 \\
\hline$\sigma_{s d+g}$ & - & - & 0.07 & 0.06 \\
\hline$\sigma_{q e}$ & 22.85 & 19.62 & 21.12 & 22.05 \\
\hline$\sigma_{q s d}$ & - & - & 1.94 & 0.84 \\
\hline$\sigma_{t s d}$ & - & - & 12.47 & 12.92 \\
\hline$\sigma_{d d}$ & - & - & 0.61 & 0.26 \\
\hline \hline $27 A l$ & & & & \\
\hline$\sigma_{t o t}$ & 697.32 & 714.35 & 675.93 & 688.08 \\
\hline$\sigma_{e l}$ & 201.02 & 212.26 & 188.22 & 196.28 \\
\hline$\sigma_{s d}$ & - & - & 10.82 & 4.56 \\
\hline$\sigma_{s d+g}$ & - & - & 0.12 & 0.11 \\
\hline$\sigma_{q e}$ & 36.39 & 31.75 & 33.24 & 34.86 \\
\hline$\sigma_{q s d}$ & - & - & 2.92 & 1.23 \\
\hline$\sigma_{t s d}$ & - & - & 19.47 & 20.42 \\
\hline$\sigma_{d d}$ & - & - & 0.91 & 0.38 \\
\hline \hline $48 T i$ & & & & 0.17 \\
\hline$\sigma_{t o t}$ & 1113.52 & 1135.53 & 1074.67 & 1095.93 \\
\hline$\sigma_{e l}$ & 353.89 & 369.77 & 327.78 & 342.93 \\
\hline$\sigma_{s d}$ & - & - & 16.86 & 6.86 \\
\hline$\sigma_{s d+g}$ & - & - & 0.17 & 0.16 \\
\hline$\sigma_{q e}$ & 48.73 & 42.82 & 44.57 & 46.86 \\
\hline$\sigma_{q s d}$ & - & - & 3.85 & 1.61 \\
\hline$\sigma_{t s d}$ & - & - & 26.11 & 27.45 \\
\hline$\sigma_{d d}$ & - & - & 1.20 & 0.50 \\
\hline & & & & \\
\hline
\end{tabular}

In our calculations nuclear densities which give the correct nuclear rms radius have adopted, and this is a reason of some differences with the results of Ref. [10] in the case of Glauber calculations. The parameters for the total nucleon-nucleon cross section and the slope of the Glauber profiles have been obtained as in Ref. [10]

\section{DIFFRACTIVE EXCITATION OF THE PROTON IN $p A$ COLLISIONS}

While the Glauber model, which is a single channel approximation, cannot go beyond elastic scattering, the dipole approach treats diagonal and off-diagonal diffractive channels on the same footing. Although the calculation of exclusive channels of diffractive excitation needs knowledge of the light-cone wave function of the final state (e.g. see [27, 28]), the total cross section of diffractive excitation summed over final states is easier to obtain, since one can employ completeness. Following the

TABLE II: HERA B (continuation of Table I)

\begin{tabular}{|c|c|c|c|c|}
\hline${ }^{{ }^{184} W}$ & Glauber & $\begin{array}{c}\text { Glauber } \\
+ \text { SRC }\end{array}$ & $\begin{array}{c}\mathrm{q}-2 \mathrm{q} \text { model } \\
+\mathrm{SRC}\end{array}$ & $\begin{array}{c}\text { 3q model } \\
+\mathrm{SRC}\end{array}$ \\
\hline$\sigma_{t o t}$ & 2972.02 & 2986.46 & 2688.09 & 2747.66 \\
\hline$\sigma_{e l}$ & 1174.09 & 1187.18 & 1025.16 & 1074.48 \\
\hline$\sigma_{s d}$ & - & - & 32.27 & 11.75 \\
\hline$\sigma_{s d+g}$ & - & - & 0.28 & 0.23 \\
\hline$\sigma_{q e}$ & 67.04 & 58.35 & 57.60 & 60.89 \\
\hline$\sigma_{q s d}$ & - & - & 4.92 & 2.04 \\
\hline$\sigma_{t s d}$ & - & - & 33.74 & 35.66 \\
\hline$\sigma_{d d}$ & - & - & 1.54 & 0.64 \\
\hline \multicolumn{5}{|l|}{\begin{tabular}{l|}
${ }^{197} A u$ \\
\end{tabular}} \\
\hline$\sigma_{t o t}$ & 2976.26 & 2989.94 & 2859.84 & 2920.75 \\
\hline$\sigma_{e l}$ & 1193.54 & 1206.10 & 1100.54 & 1150.99 \\
\hline$\sigma_{s d}$ & - & - & 32.55 & 11.95 \\
\hline$\sigma_{s d+g}$ & - & - & 0.29 & 0.24 \\
\hline$\sigma_{q e}$ & 62.94 & 54.69 & 61.15 & 64.53 \\
\hline$\sigma_{q s d}$ & - & - & 5.31 & 2.22 \\
\hline$\sigma_{t s d}$ & - & - & 35.82 & 37.80 \\
\hline$\sigma_{d d}$ & - & - & 1.66 & 0.69 \\
\hline \multicolumn{5}{|l|}{${ }^{208} \mathrm{~Pb}$} \\
\hline$\sigma_{t o t}$ & 3052.11 & 3117.62 & 2955.57 & 3018.21 \\
\hline$\sigma_{e l}$ & 1243.00 & 1274.60 & 1147.01 & 1199.14 \\
\hline$\sigma_{s d}$ & - & - & 32.88 & 12.02 \\
\hline$\sigma_{s d+g}$ & - & - & 0.29 & 0.24 \\
\hline$\sigma_{q e}$ & 62.55 & 54.11 & 61.01 & 64.39 \\
\hline$\sigma_{q s d}$ & - & - & 5.31 & 2.21 \\
\hline$\sigma_{t s d}$ & - & - & 35.73 & 37.71 \\
\hline$\sigma_{d d}$ & - & - & 1.66 & 0.69 \\
\hline
\end{tabular}

standard classification of diffractive channels in terms of the triple Regge approach [29], one can consider diffractive excitation of the valence quark system, which corresponds to the Pomeron-Pomeron-Reggeon $(\mathbb{P} \mathbb{P} \mathbb{R})$ term, and diffractive gluon radiation corresponding to the triple Pomeron term $(\mathbb{P} \mathbb{P} \mathbb{P})$. The former mostly contributes to small mass excitations, $d \sigma / d M_{X}^{2} \propto 1 / M_{X}^{3}$, while the latter is responsible for the large mass tail, $d \sigma / d M_{X}^{2} \propto$ 
$1 / M_{X}^{2}$, where $M_{X}$ is the invariant mass of the produced system, $p p \rightarrow X p$.

\section{A. Coherent excitation of the projectile valence quark system}

The cross section of coherent single diffraction on a nucleus, caused by excitation of the valence quark skeleton without gluon radiation, is given as usual by the dispersion of the distribution of eigen elastic amplitudes, where the eigenstates are the dipoles [5, 26].

$$
\begin{gathered}
\sigma_{s d}(p A \rightarrow X A) \mathbb{P} \mathbb{P} \mathbb{R}=\int d^{2} b e^{I_{A}(b)} \\
\times\left[\left\langle e^{-\sigma_{d i p} T_{A}^{h}(b)}\right\rangle-\left\langle e^{-\frac{1}{2} \sigma_{d i p} T_{A}^{h}(b)}\right\rangle^{2}\right],
\end{gathered}
$$

where $I_{A}(b)$ is given by Eq. (55). Dependent on the model, the dipole cross section here has the form of either Eq. (36), or (49), and the averaging is weighed by the wave function squared having the form of either Eq. (38), or (48).

TABLE III: RHIC

\begin{tabular}{c|cccc}
\hline \hline${ }^{12} C$ & Glauber & $\begin{array}{c}\text { Glauber } \\
+ \text { SRC }\end{array}$ & $\begin{array}{c}\text { q-2q model } \\
+ \text { SRC }\end{array}$ & $\begin{array}{c}\text { 3q model } \\
+ \text { SRC }\end{array}$ \\
\hline$\sigma_{t o t}$ & 413.71 & 425.73 & 406.90 & 410.20 \\
\hline$\sigma_{e l}$ & 112.13 & 119.68 & 109.16 & 111.29 \\
\hline$\sigma_{s d}$ & - & - & 3.13 & 1.20 \\
\hline$\sigma_{s d+g}$ & - & - & 0.31 & 0.30 \\
\hline$\sigma_{q e}$ & 26.40 & 23.09 & 26.13 & 26.72 \\
\hline$\sigma_{q s d}$ & - & - & 0.95 & 0.29 \\
\hline$\sigma_{t s d}$ & - & - & 10.90 & 11.14 \\
\hline$\sigma_{d d}$ & - & - & 0.95 & 0.29 \\
\hline \hline $208 P b$ & & & & \\
\hline$\sigma_{t o t}$ & 3297.56 & 3337.57 & 3228.11 & 3262.58 \\
\hline$\sigma_{e l}$ & 1368.36 & 1398.08 & 1314.04 & 1343.76 \\
\hline$\sigma_{s d}$ & - & - & 16.78 & 5.03 \\
\hline$\sigma_{s d+g}$ & - & - & 1.06 & 0.98 \\
\hline$\sigma_{q e}$ & 66.06 & 58.47 & 71.99 & 73.92 \\
\hline$\sigma_{q s d}$ & - & - & 2.39 & 0.56 \\
\hline$\sigma_{t s d}$ & - & - & 30.03 & 30.83 \\
\hline$\sigma_{d d}$ & - & - & 2.39 & 0.56 \\
\hline \hline & & & & \\
\hline \hline
\end{tabular}

Although Gribov corrections to the total cross section are known to be small, well within $10 \%$ [30, 31], this is because they affect only the second exponential term in Eq. (8), which is small. However, this term itself is modified significantly by the inelastic shadowing corrections. Therefore, one should expect a considerable increase of both terms in (57) due to inelastic corrections, which make the nuclear medium considerably more transparent compared to the Glauber model [5]. Nevertheless, both terms are small for heavy nuclei and suppress diffraction everywhere except at the nuclear periphery. Thus, the cross section of single diffraction should rise as $A^{1 / 3}$, with a coefficient which is sensitive to the inelastic shadowing corrections and $N N$ correlations.

The details of the calculations with both models under consideration can be found in [10]. The numerical results for several nuclei and energies are presented in Tables I, II, III and IV.

\begin{tabular}{|c|c|c|c|c|}
\hline${ }^{12} \mathrm{C}$ & Glauber & $\begin{array}{c}\text { Glauber } \\
+\mathrm{SRC}\end{array}$ & $\begin{array}{c}\mathrm{q}-2 \mathrm{q} \text { model } \\
+\mathrm{SRC}\end{array}$ & $\begin{array}{c}3 \mathrm{q} \text { model } \\
+\mathrm{SRC}\end{array}$ \\
\hline$\sigma_{t o t}$ & 598.79 & 613.68 & 591.05 & 592.12 \\
\hline$\sigma_{e l}$ & 198.11 & 208-59 & 194.84 & 195.65 \\
\hline$\sigma_{s d}$ & - & - & 0.74 & 0.20 \\
\hline$\sigma_{s d+g}$ & - & - & 2.58 & 2.56 \\
\hline$\sigma_{q e}$ & 49.10 & 45.42 & 45.03 & 45.22 \\
\hline$\sigma_{q s d}$ & - & - & -0.66 & -0.86 \\
\hline$\sigma_{t s d}$ & - & - & 6.97 & 7.00 \\
\hline$\sigma_{d d}$ & - & - & -0.66 & -0.86 \\
\hline \multicolumn{5}{|l|}{${ }^{208} \mathrm{~Pb}$} \\
\hline$\sigma_{t o t}$ & 3850.63 & 3885.77 & 3833.26 & 3839.26 \\
\hline$\sigma_{e l}$ & 1664.76 & 1690.48 & 1655.70 & 1660.67 \\
\hline$\sigma_{s d}$ & - & - & 2.62 & 0.59 \\
\hline$\sigma_{s d+g}$ & - & - & 2.58 & 2.56 \\
\hline$\sigma_{q e}$ & 120.92 & 112.65 & 113.37 & 113.88 \\
\hline$\sigma_{q s d}$ & - & - & -2.08 & -2.62 \\
\hline$\sigma_{t s d}$ & - & - & 17.55 & 17.63 \\
\hline$\sigma_{d d}$ & - & - & -2.08 & -2.62 \\
\hline
\end{tabular}

TABLE IV: LHC

\section{B. Coherent diffractive gluon radiation}

Diffractive gluon radiation also contributes to the single diffractive process $p A \rightarrow X A$. Correspondingly, the single-diffraction cross section Eq. (57) must be corrected for this excitation channel. The cross section of coherent gluon radiation on a nucleus is given by [33],

$$
\begin{aligned}
& \sigma_{s d}(p A \rightarrow X A)_{3 \mathbb{P}}=\frac{3}{4 \pi} \ln \left[\frac{s\left(1-x_{0}\right)}{M_{0}^{2}}\right] \\
\times & \int d^{2} b e^{I_{A}(b)}\left\langle e^{-\frac{1}{2} \sigma_{d i p} T_{A}^{h}}\right\rangle^{2} \int d^{2} r_{T}\left|\Psi_{q G}\left(\vec{r}_{T}\right)\right|^{2} \\
\times & \left\{1-\exp \left[-\frac{9}{16}\left(\sigma_{\bar{q} q}\left(r_{T}, s\right) T_{A}^{h}(b)-\frac{9}{8} I_{A}(b)\right)\right]\right\}^{2}(58)
\end{aligned}
$$

Here $M_{0}^{2}=5 \mathrm{GeV}^{2}$ is the minimal effective mass squared of the proton excitation, $x_{0}=0.85$ is the minimal value of Feynman $x$, which can be treated as being in the domain of the triple-Regge kinematics. [29]. 
The first factor in (58) accounts for the absorptive corrections, which are due to the lack of initial/final state interaction of the valence quarks propagating through the nucleus. Further details about the calculations can be found in [10]. The numerical results for several nuclei and energies are presented in Tables I, II, III and IV.

\section{QUASIELASTIC SCATTERING WITH AND WITHOUT EXCITATION OF THE PROJECTILE}

In the cases when either the beam proton $(p A \rightarrow X A)$, or the nucleus $\left(p A \rightarrow p A^{*}\right)$, or both $\left(p A \rightarrow X A^{*}\right)$, are diffractively excited, one can make use of completeness, which substantially simplifies the calculations. As was already mentioned, the important condition for the nucleus is that it decays into nuclear fragments with no new particle produced. The dipole formalism for these processes was developed in [10]. Here we rely on those results and introduce corrections related to $N N$ correlations.

The simplest processes are double excitation $p A \rightarrow$ $X A^{*}$, where $X$ includes the ground state proton, as well as quasi-diffraction, with all diffractive excitation of its valence quark system (without gluon radiation), and breakup of the nucleus. All channels of coherent interactions which leave the nucleus intact, should be subtracted,

$$
\begin{aligned}
& \sigma_{q e l}\left(p A \rightarrow p A^{*}\right)+\sigma_{q s d}\left(p A \rightarrow X A^{*}\right) \\
= & \int d^{2} b\left\langle e^{-\sigma_{\text {dip }} T_{A}^{h}(b)}\left\{e^{\tilde{I}_{A}(b)} e^{\frac{\sigma_{d i p}^{2} T_{A}^{h}(b)}{16 \pi B_{e l}}}-e^{I_{A}(b)}\right\}\right\rangle \\
= & \int d^{2} b \sum_{k=0} \frac{1}{k !}\left[\frac{T_{A}^{h}(b)}{16 \pi B_{e l}}\right]^{k}\left[e^{\tilde{I}_{A}(b)}-e^{I_{A}(b)} \delta_{k 0}\right] \\
\times & \frac{\partial^{2 k}}{\partial\left(T_{A}^{h}\right)^{2 k}}\left\langle e^{-\sigma_{\text {dip }} T_{A}^{h}(b)}\right\rangle .
\end{aligned}
$$

Here besides the function $I_{A}(b)$ defined in (55) we introduce a new one,

$$
\begin{aligned}
& \tilde{I}_{A}(b)=\int d^{2} l_{1} d^{2} l_{2} \Delta_{A}^{\perp}\left(\vec{l}_{1}, \vec{l}_{2}\right) \\
\times & \left\langle\left[2 \operatorname{Re} \Gamma^{\bar{q} q}\left(\vec{l}_{1}, \vec{r}_{T}, \alpha\right)-\left(\operatorname{Re} \Gamma^{\bar{q} q}\left(\vec{l}_{1}, \vec{r}_{T}, \alpha\right)\right)^{2}\right]\right. \\
\times & {\left.\left[2 \operatorname{Re} \Gamma^{\bar{q} q}\left(\vec{l}_{2}, \vec{r}_{T}, \alpha\right)-\left(\operatorname{Re} \Gamma^{\bar{q} q}\left(\vec{l}_{2}, \vec{r}_{T}, \alpha\right)\right)^{2}\right]\right\rangle } \\
\approx & {\left[\frac{\sigma_{t o t}^{p N}-\sigma_{e l}^{p N}-\sigma_{s d}^{p N}}{\sigma_{\text {tot }}^{p N}}\right]^{2} I_{A}(b) }
\end{aligned}
$$

In order to simplify the calculations, we neglect here the difference in the slopes of powers of the partial amplitude. This is a second order correction, i.e. a correction to a correction.

One can single out in (59) the quasielastic channel. For that purpose one should average over the dipole sizes, separately for both the incoming and outgoing protons,

$$
\begin{aligned}
\sigma_{q e l}^{p A} & =\int d^{2} b\left\langle\left\langle e^{-\frac{1}{2} \sigma_{d i p}^{(1)} T_{A}^{h}(b)} e^{-\frac{1}{2} \sigma_{d i p}^{(2)} T_{A}^{h}(b)}\right.\right. \\
& \left.\left.\times\left[e^{\tilde{I}_{A}(b)} e^{\frac{1}{16 \pi B_{e l}} \sigma_{d i p}^{(1)} \sigma_{d i p}^{(2)} T_{A}^{h}(b)}-e^{I_{A}(b)}\right]\right\rangle_{1}\right\rangle_{2} \\
& =\int d^{2} b \sum_{k=0} \frac{1}{k !}\left[\frac{T_{A}^{h}(b)}{4 \pi B_{e l}}\right]^{k}\left[e^{\tilde{I}_{A}(b)}-e^{I_{A}(b)} \delta_{k 0}\right] \\
& \times\left\{\frac{\partial^{k}}{\partial\left(T_{A}^{h}\right)^{k}}\left\langle e^{-\frac{1}{2} \sigma_{d i p} T_{A}^{h}(b)}\right\rangle\right\}^{2} .
\end{aligned}
$$

This is a fast converging series due to the smallness of the elastic cross section. We control the accuracy to be within $1 \%$.

Subtracting (61) from (59) one can get the quasidiffractive cross section, which includes the proton excitations without gluon radiation. To include gluon radiation we use the same prescription as in (54), replacing the $\mathbb{P} \mathbb{P} \mathbb{R}$ term, $\left[\sigma_{s d}^{p p}\right]_{\mathbb{P} \mathbb{P} \mathbb{R}}$, by the total single-diffraction cross section.

In the case of nuclear breakup the recoil bound nucleon can be also diffractively excited. We relate the cross sections for such channels to the above calculated quasi-elastic and quasi-diffractive processes, in the same way as in [10].

\section{GLUON SHADOWING}

In terms of the parton model, gluon shadowing is interpreted in the nuclear infinite momentum frame as a result of fusion of gluons originating from different bound nucleons. This process leads to a reduction of the gluon density in the nucleus at small $x$. The ultimate form of gluon shadowing is gluon saturation [32].

In terms of the dipole approach gluon shadowing is described as Glauber shadowing for higher Fock states containing gluons, [33]. The effect turns out to be rather weak due to the shortness of the quark-gluon and gluongluon correlation radius, an observation which is supported by many experimental evidences [34, 35]. For this reason we neglect the small effects of nucleon correlations in the calculation of gluon shadowing, and use the results of Ref. [10].

\section{CONCLUSIONS}

In this paper we further developed the dipole approach of [5, 9, 10] to the calculation of Gribov inelastic corrections. We employed two models for the proton wave function, which result in reasonable diffractive cross sections for $p p$ collisions. Here we increased the accuracy of the calculation of the cross sections of different diffractive processes on nuclei by improving the model for the nuclear wave function. Namely, we went beyond the popular single particle density approximation and introduced 
corrections for nucleon-nucleon correlations, which lead to sizable effects, modifying the effective nuclear thickness function [12]. While inelastic shadowing corrections make the nuclear medium more transparent for colorless hadrons, the nucleon short range correlations work in the opposite direction making the medium more opaque. The influence of both effects on different diffractive channels vary. They are especially large for quasielastic and quasisingle diffractive processes associated with the survival probability of colorless hadrons propagating through a nuclear medium.

\section{Acknowledgments}

This work was supported in part by Fondecyt (Chile) grants 1090236 and 1090291, and by DFG (Germany) grant PI182/3-1. C.d.A. thanks HELEN project for support during his visit to the Department of Physics, UTFSM, Valparaiso, where this work has been initiated. M.A. was supported by a DOE grant under contract DE-FG02-93ER40771, he also thanks HPC-EUROPA2 (project number: 228398) with the support of the EU Research Infrastructure Action FP7. This work was finished during an extended visit by the authors at INT, University of Washington; they are thankful to the INT staff for support and warm hospitality.
[1] R. J. Glauber, in Lectures in Theoretical Physics, W. E. Brittin et al Editors, New York (1959).

[2] V. N. Gribov, Sov. JETP 29 (1969) 483. J. Pumplin, M. Ross, Phys. Rev. Lett. 21 (1968) 1778.

[3] V. Karmanov and L.A. Kondratyuk, Sov. Phys. JETP Lett. 18 (1973) 266.

[4] B. Z. Kopeliovich and L. I. Lapidus, Pisma Zh. Eksp. Teor. Fiz. 28, 664 (1978).

[5] B. Z. Kopeliovich, L. I. Lapidus and A. B. Zamolodchikov, JETP Lett. 33, 595 (1981) [Pisma Zh. Eksp. Teor. Fiz. 33, 612 (1981)].

[6] B.Z. Kopeliovich, J. Raufeisen and A.V. Tarasov, Phys. Lett. B440 (1998) 151.

[7] B.Z. Kopeliovich, J. Raufeisen and A.V. Tarasov, Phys. Rev. C62 (2000) 035204.

[8] B. Z. Kopeliovich, I. Schmidt and M. Siddikov, arXiv:0906.5589 [hep-ph], to appear in Phys. Rev. D.

[9] B.Z. Kopeliovich, Phys. Rev. C68 (2003) 044906.

[10] B. Z. Kopeliovich, I. K. Potashnikova and I. Schmidt, Phys. Rev. C 73, 034901 (2006).

[11] R. Subedi et al, Science 320, 1476 (2008). E. Piazetsky, M. Sargsian, L. Frankfurt, M. Strikman and J, W. Watson Phys. Rev. Lett. 97 (2006) 162504.

A. Tang et al, Phys. Rev. Lett. 90 (2003) 042301.

R. Shneor et al, Phys. Rev. Lett., 99 (2007) 072501.

[12] M. Alvioli, C. Ciofi degli Atti, H. Morita, V. Palli Phys. Rev.C78 (2008) 031601(R).

[13] L. L. Foldy and J. D. Walecka, Ann. Phys. 54, (1969) 447.

[14] M. Alvioli, C. Ciofi degli Atti, H. Morita, V. Palli, in preparation.

[15] M. Alvioli, C. Ciofi degli Atti, H. Morita, Phys. Rev. C72 (2005) 054310.

[16] M. Alvioli, C. Ciofi degli Atti, H. Morita, Phys. Rev. Lett.100 (2008) 162503.

[17] B. S. Pudliner, V. R. Pandharipande, J. Carlson, S. C. Pieper and R. B. Wiringa, Phys. Rev. C56, (1997) 1720.

[18] H. De Vries, C. W. De Jager and C. De Vries, Nucl. Data.
Tables 36 495(1987);

[19] S. Amendolia et al., Nucl. Phys. B277 (1986) 186.

[20] I.A. Schmidt and R. Blankenbecler, Phys. Rev. D 15 , 3321 (1977).

[21] M. Anselmino, E. Predazzi, S. Ekelin, S. Fredriksson, and D.B. Lichtenberg, Rev. Mod. Phys. 65 (1993) 1199.

[22] B.Z. Kopeliovich and B.G. Zakharov, Phys. Lett. 211B (1988) 221; Sov. J. Nucl. Phys. 48 (1988) 136; ibid 49 (1989) 674; Z. Phys. C43 (1989) 241; Sov. Phys. Particles and Nuclei, 22 (1991) 140.

[23] B. Z. Kopeliovich, H. J. Pirner, A. H. Rezaeian and I. Schmidt, Phys. Rev. D 77, 034011 (2008).

[24] B. Z. Kopeliovich, I. K. Potashnikova, I. Schmidt and J. Soffer, Phys. Rev. D 78, 014031 (2008).

[25] B. Z. Kopeliovich, A. H. Rezaeian and I. Schmidt, Phys. Rev. D 78, 114009 (2008).

[26] B. Z. Kopeliovich, I. K. Potashnikova and I. Schmidt, Braz. J. Phys. 37, 473 (2007) [arXiv:hep-ph/0604097].

[27] Yu. P. Ivanov, B. Z. Kopeliovich, A. V. Tarasov and J. Hufner, Phys. Rev. C 66, 024903 (2002).

[28] B. Z. Kopeliovich, J. Nemchik, A. Schafer and A. V. Tarasov, Phys. Rev. C 65, 035201 (2002).

[29] Yu.M. Kazarinov, B.Z. Kopeliovich, L.I. Lapidus and I.K. Potashnikova, JETP 70 (1976) 1152.

[30] P.V.R. Murthy et al., Nucl. Phys. B92, 269 (1975).

[31] A. Gsponer et al., Phys. Rev. Lett. 42, 9 (1979).

[32] L.V. Gribov, E.M. Levin and M.G. Ryskin, Nucl. Phys. B188 (1981) 555; Phys. Rep. 100 (1983) 1.

[33] B.Z. Kopeliovich, A. Schäfer and A.V. Tarasov, Phys. Rev. D62 (2000) 054022.

[34] B.Z. Kopeliovich and B. Povh, J. Phys. G30, S999 (2004).

[35] B. Z. Kopeliovich, I. K. Potashnikova, B. Povh and I. Schmidt, Phys. Rev. D 76, 094020 (2007)

[36] We ignore the effect of motion of the center of gravity assuming the nucleus to be sufficiently heavy. 